\title{
Fixing Idioms
}

\section{A recursion primitive for applicative DSLs}

\author{
Dominique Devriese Ilya Sergey Dave Clarke Frank Piessens \\ iMinds-DistriNet, KU Leuven \\ \{firstname.lastname\}@cs.kuleuven.be
}

\begin{abstract}
In a lazy functional language, the standard encoding of recursion in DSLs uses the host language's recursion, so that DSL algorithms automatically use the host language's least fixpoints, even though many domains require algorithms to produce different fixpoints. In particular, this is the case for DSLs implemented as Applicative functors (structures with a notion of pure computations and function application). We propose a recursion primitive afix that models a recursive binder in a finally tagless HOAS encoding, but with a novel rank-2 type that allows us to specify and exploit the effectsvalues separation that characterises Applicative DSLs. Unlike related approaches for Monads and Arrows, we model effectful recursion, not value recursion.

Using generic programming techniques, we define an aritygeneric version of the operator to model mutually recursive definitions. We recover intuitive user syntax with a form of shallow syntactic sugar: an alet construct that syntactically resembles the let construct, which we have implemented in the GHC Haskell compiler. We describe a proposed axiom for the afix operator. We demonstrate usefulness with examples from Applicative parser combinators and functional reactive programming. We show how higher-order recursive operators like many can be encoded without special library support, unlike previous approaches, and we demonstrate an implementation of the left recursion removal transform.
\end{abstract}

Categories and Subject Descriptors $\quad$ D.3.3 [Language Constructs and Features]: Recursion

Keywords Applicative functors, observable recursion, HOAS

\section{Introduction}

Let us start with an embedded domain-specific language (EDSL) of parser rules, modelled as the GADT (see e.g. |29|) Rule. The data type is parameterised by the type $a$ of parse results:

$$
\begin{aligned}
& \text { data Rule } a \text { where } \\
& \text { Pure }:: a \rightarrow \text { Rule } a \\
& \text { Seq }:: \text { Rule }(a \rightarrow b) \rightarrow \text { Rule } a \rightarrow \text { Rule } b \\
& \text { Disj } \quad:: \text { Rule } a \rightarrow \text { Rule } a \rightarrow \text { Rule } a
\end{aligned}
$$

Permission to make digital or hard copies of all or part of this work for personal or classroom use is granted without fee provided that copies are not made or distributed for profit or commercial advantage and that copies bear this notice and the full citation on the first page. To copy otherwise, to republish, to post on servers or to redistribute to lists, requires prior specific permission and/or a fee.

PEPM'13, January 21-22, 2013, Rome, Italy.

Copyright (C) 2013 ACM 978-1-4503-1842-6/13/01 . . \$15.00

\author{
Fail :: Rule a \\ Token :: Char $\rightarrow$ Rule Char
}

Rule provides DSL primitives Pure (match the empty string, return a fixed result), $S e q$ (sequence two rules, apply the result of the first to that of the second), Disj (choose between two rules), Fail and Token (parse and return a specified character).

Rule uses the Applicative parser combinator style introduced and popularised by Swierstra et al. [31. Readers may recognise $S e q$ and Pure as pure and $\circledast$ operators of an Applicative functor. With Haskell's $a^{\prime} f^{\prime} b$ for $f a b$, the following $b s$ and $b s^{\prime}::$ Rule String model a language of arbitrary-length sequences of bs:

$$
\begin{aligned}
& \text { bs }=(\text { Pure (:) 'Seq' Token 'b' 'Seq' bs) 'Disj' Pure " " } \\
& b s^{\prime}=(\text { Pure snoc 'Seq' bs' 'Seq' Token 'b') 'Disj' Pure " " }
\end{aligned}
$$

According to rule $b s$, matches either start with token ' $\mathrm{b}$ ', followed by another match of $b s$ or they are empty. The rule Pure (:) has no parse behaviour of $b s$, but produces the list cons operator $(:)$ as a result, so that the parsed token ' $b$ ' is consed with the result of the recursive match. For an empty match, the empty string " " is returned. Parser rule $b s^{\prime}$ defines the same language and parse results but expects the recursive match first, and the token 'b' second. $b s^{\prime}$ is left-recursive: it refers to itself in a left-most position.

The algorithm nullable :: Rule $a \rightarrow$ Bool checks if a rule accepts the empty string:

$$
\begin{aligned}
& \text { nullable }\left(\text { Pure } \_\right)=\text {True } \\
& \text { nullable }(\text { Seq } a b)=\text { nullable } a \wedge \text { nullable } b \\
& \text { nullable }(\text { Disj } a b)=\text { nullable } a \vee \text { nullable } b \\
& \text { nullable Fail = False } \\
& \text { nullable }(\text { Token _ })=\text { False }
\end{aligned}
$$

This definition is satisfactory for finite rules, but a problem arises for infinite, recursive production rules like $b s$ and $b s^{\prime}$ above.

A known problem of parser DSLs like Rule is that left-recursive rules are not treated well. Unlike nullable bs (which is True), nullable $b s^{\prime}$ is $\perp$ : computation loops forever. Computationally, nullable $b s^{\prime}$ loops when considering the left-most part of its first alternative. Denotationally, nullable $b s^{\prime}$ corresponds to the fixpoint of a certain function, and the least fixpoint $\perp$ we get from Haskell is not the one we would like.

This example shows that for DSLs like our grammar model, algorithms need more control of how fixpoints are calculated. As such, it is inappropriate to rely on Haskell's least fixpoints. Otherwise, parsing libraries are restricted to top-down parsing algorithms, leftrecursion is difficult (although some algorithms deal with it anyway, e.g. [16]) and some algorithms are impossible (e.g. print a representation of a rule's parsing structure). Also for DSLs with re- 
cursion in other domains, useful analyses and transformations are rendered impossible by the implicit or unobservable recursion.

\subsection{Overview and Contributions}

Like Oliveira and Cook [25], we propose to represent recursive definitions using a recursive binder $\mu$, e.g.:

$$
\text { bs }=\mu \text { s. (Pure (:) 'Seq' Token 'b' 'Seq's) 'Disj' Pure " " }
$$

After some more background in section 2 we encode such a primitive in HOAS style in section 3 We don't use standard HOAS as it allows certain illegal terms, but use Carette et al.'s finally tagless style instead. We adapt the technique from lambda terms to applicative DSLs (no Lambda binder but a pure primitive instead) with a new representation of the recursive binder: the afix primitive.

$$
\begin{aligned}
& \text { class Applicative } p \Rightarrow \text { ApplicativeFix } p \text { where } \\
& \text { afix }::(\forall q \text {. Applicative } q \Rightarrow p(q a) \rightarrow p(q a)) \rightarrow p a
\end{aligned}
$$

The primitive differs from Carette et al.'s Lambda because of its rank-2 type. We show how it models a separation between values and effects of the DSL. This reflects the separation that distinguishes applicative DSLs from monadic ones and makes them suited for analysis and optimisation. In section 4 , we discuss an axiom that governs the intended semantics (recursion) of afix.

A downside of an explicit fixpoint primitive like $a f i x$ is that we lose some of the elegance of Haskell's standard recursive equations. In section 5. we define a shallow form of syntactic sugar to recover Haskell's standard elegance in the form of the alet 11 construct, e.g.:

$$
\text { alet } b s=(:) \text { (token ' } \mathrm{b} \text { ' } \circledast b s \oplus \text { pure " " }
$$

We define scoping and typing rules for alet and a desugaring into standard Haskell, all implemented in our branch of the Glasgow Haskell Compiler (GHC). Using generic programming techniques, we implement a mutual recursion primitive that reduces mutually recursive bindings to a series of recursive bindings.

Finally, in section 6 we demonstrate the usefulness of our techniques with two larger examples. One example concerns a simple functional reactive programming (FRP) model of electronic hardware and the second example shows a non-trivial parser transformation that removes left-recursion from a parser.

\section{Background, examples and machinery}

\subsection{More fixpoints}

Consider how $c b e^{\prime}=$ nullable $b s^{\prime}$ produces $\perp$ as the wrong solution of a recursive equation. Denotationally (see e.g. [36]), $c b e^{\prime}=\perp$ is the least fixpoint of $c b e f^{\prime}$ under the standard complete partial order on values ${ }^{2}$

$$
\text { cbef }{ }^{\prime} s=(s \wedge \text { False }) \vee \text { True }
$$

The fact that this least fixpoint is $\perp$ is especially unfortunate because $c b e f^{\prime}$ does have a non- $\perp$ fixpoint: $c b e f^{\prime}$ True $\equiv$ True.

Based on nullable, we can also calculate the first set of a rule: the set of characters that can start a successful parse.

$$
\begin{aligned}
& \text { firstSet }:: \text { Rule } a \rightarrow \text { Set Char } \\
& \text { firstSet }(\text { Pure }-) \quad \text { Set.empty }
\end{aligned}
$$

\footnotetext{
${ }^{1}$ The word "applet" was already taken...

${ }^{2}$ Note: this is not the standard meaning of $c b e^{\prime}$ but it can be shown because of the structure of nullable and $b s^{\prime}$.
}

firstSet $($ Seq $a b)=$

if nullable $a$ then firstSet $a \cup$ firstSet $b$ else firstSet $a$ firstSet $($ Disj $a b)=$ firstSet $a \cup$ firstSet $b$

firstSet Fail = Set.empty

firstSet $($ Token $t)=$ singleton $t$

If nullable $b s^{\prime}$ were True instead of $\perp$, then $f s^{\prime}=$ firstSet $b s^{\prime}$ also gives us the least fixpoint of a function $f s f^{\prime}$ :

$$
f_{s} f^{\prime} s=(s \cup \text { singleton ' } \mathrm{b} \text { ' }) \cup \text { Set.empty }
$$

$f_{s f} f^{\prime}$ has several fixpoints: singleton ' $\mathrm{b}$ ', but also $\perp$ (chosen by Haskell) and Set.fromList "abc". Only one corresponds to intuition and language theory: singleton ' $\mathrm{b}$ '. To conclude, algorithm authors should use domain knowledge to choose appropriate fixpoints and the representation of recursion should support this.

\subsection{Applicative Functors}

Rule's constructors fit the pattern of an Applicative functor [23], a type class modelling structures with a notion of pure computations (pure) and application $(\circledast)]^{3}$

class Functor $p \Rightarrow$ Applicative $p$ where

pure :: $a \rightarrow p$ a

$(\circledast):: p(a \rightarrow b) \rightarrow p a \rightarrow p b$

instance Applicative Rule where

pure $=$ Pure

$(\circledast)=S e q$

Rule also instantiates Alternative: a class of Applicative functors with a notion of disjunction (D) and failure (empty).

class Applicative $p \Rightarrow$ Alternative $p$ where

(Ф) $\quad:: p a \rightarrow p a \rightarrow p a$

empty :: $p$ a

instance Alternative Rule where ...

\subsection{Applicative functors vs. Monads}

The reason we work with Applicative DSLs and not for example the better known Monads is that for an analysis to handle recursive equations (our main goal), it must be able to observe the structure of the recursive equation. Monadic DSLs use a monadic bind $\gg$ typed $m a \rightarrow(a \rightarrow m b) \rightarrow m b$ that allows the effects structure of a term to depend on the result value of a previous term, rendering the computation impossible to analyse. Consider the term

$$
\begin{aligned}
\text { evilMonadic }=\text { do } & n \leftarrow \text { evilMonadic } \\
& \text { if } n \equiv 100 \text { then evilMonadic } \\
& \text { else return }(100-n)
\end{aligned}
$$

It is unclear if this recursive equation has a non- $\perp$ solution, but even if it has, it is hopeless to find it. The do-notation translates to evilMonadic $\gg(\lambda n \rightarrow$ if $n \equiv 100 \cdots)$ and this second argument is a function that may return entirely different rules for different $n$. The monadic bind can not inspect all cases, so that finding non-least fixpoints is hopeless in all but the simplest cases.

Unlike $\gg$, the Applicative apply operator $\circledast$ 's type $(p \quad(a \rightarrow$ $b) \rightarrow p a \rightarrow p \quad b)$ prevents computations' values from influencing the structure of subsequent computations. This separates a computation's effects from its values, corresponding for parsers to context-free-ness (roughly, see [21]). This makes Applicative parser libraries well-suited for analysis and optimisation [1, 31] and makes the goal of finding non-least fixpoints feasible.

\footnotetext{
${ }^{3}$ We consistently omit Functor instances because its fmap or $\$$ method should satisfy $f \circledast p=$ pure $f \circledast p$.
} 


\subsection{Composing Applicative functors}

An important tool for us is the composition of two Applicative functors $p$ and $q[23]$. The composed functor $(p \circ q)$ is again Applicative.

$$
\begin{gathered}
\text { newtype }(p \circ q) \text { a Comp }\{\text { comp }:: p(q a)\} \\
\text { instance }(\text { Applicative } p \text {, Applicative } q) \Rightarrow \\
\text { Applicative }(p \circ q) \text { where } \\
\text { pure }=\text { Comp } \cdot \text { pure } \cdot \text { pure } \\
f \circledast v=\text { Comp } \$(\circledast) \text { comp } f \circledast \text { comp } v
\end{gathered}
$$

For a composed functor $(p \circ q)$, we call $p$ and $q$ the outer resp. inner functor. Terms in either functor can be lifted to $(p \circ q)$. Also useful is a function withInner, lifting operations on the inner functor $q$ :

$$
\begin{gathered}
\text { liftOuter }:: \text { (Functor } p, \text { Applicative } q) \Rightarrow p a \rightarrow(p \circ q) a \\
\text { liftInner :: Applicative } p \Rightarrow q a \rightarrow(p \circ q) a \\
\text { withInner :: Functor } p \Rightarrow(q a \rightarrow h a) \rightarrow \\
\qquad(p \circ q) a \rightarrow(p \circ h) a
\end{gathered}
$$

\subsection{Effectful recursion, not value recursion}

We study DSL terms defined as the solution of recursive equations, like the examples $b s$ and $b s^{\prime}$. This is a different kind of recursion than the value recursion studied by Erkök and Launchbury [14]. They study monads that support recursive values in the parameters and results of effectful computations. For example, GHC's Data.IORef (a mutable references interface in the $I O$ Monad) supports the cells' values to be defined lazily. Using the recursive do syntax of GHC's DoRec extension (evolved from the original proposal), a function can create mutually recursive reference cells:

$$
\begin{gathered}
\text { data Node }=\text { Node Int }(\text { IORef Node }) \\
\text { mk2nodes = do rec } p \leftarrow \text { newIORef }(\text { Node } 0 r) \\
r \leftarrow \text { newIORef }(\text { Node } 1 p) \\
\text { return } p
\end{gathered}
$$

Value recursion is only interesting in the context of monadic computations. For Applicative functors, the separation of values and effects limits the value recursion entirely to the value level. The same effect can then be achieved by using the standard fix at the value level (see section 4.2). It is the monadic interplay of effects and values that makes value recursion interesting. In this paper, we study effectful recursion, a different form of recursion that is more naturally associated with applicative functors. Effectful recursion is used to define effectful recursive DSL terms, like $b s$ and $b s^{\prime}$.

\section{A recursion primitive}

Let us define our recursion primitive $a f i x$. We start from more naive definitions and iteratively adapt it to satisfy our requirements.

In our Applicative Rule DSL, we might represent a recursive binder in a naive HOAS style with a new Rule primitive FixO:

$$
\begin{aligned}
& \text { Fix0 }::(\text { Rule } a \rightarrow \text { Rule } a) \rightarrow \text { Rule } a \\
& b s=\text { Fixo } \$ \lambda b s_{0} \rightarrow(:) \text { Token ' } \mathrm{b} \text { ' } \circledast b s_{0} \text { (1) pure " " } \\
& b s^{\prime}=\text { Fixo } \$ \lambda b s_{0}^{\prime} \rightarrow \text { snoc }\left(b s_{0}^{\prime} \circledast\right. \text { Token 'b' (1) pure " " }
\end{aligned}
$$

Unfortunately, this representation allows meaningless definitions:

$$
\begin{aligned}
\text { badBind }=\text { Fixo } \$ \lambda \text { self } \rightarrow \text { case } \text { self of Pure } & \rightarrow \text { self } \\
- & \rightarrow \text { Pure } 0
\end{aligned}
$$

badBind treats its recursive parameter non-parametrically and does not model a usage of the recursive binder $\mu$. Such definitions are not excluded by FixO's type. Chlipala explains how this complicates DSL algorithms like nullable, requiring an inverse algorithm mapping result values (here: booleans) back to DSL terms [6].

We use Carette et al.'s finally tagless style [5] to solve this. Chlipala's alternative Parametric HOAS [6] is discussed in section 7 .

\subsection{Finally tagless style (second attempt)}

In Carette et al.'s finally tagless style, a parser rule with result type $a$ is not a value of data type Rule $a$, but of type FinalRule 0 :

$$
\begin{aligned}
& \text { class Applicative } p \Rightarrow \text { CharParser } p \text { where } \\
& \text { token }:: \text { Char } \rightarrow p \text { Char } \\
& \text { type FinalRule0 } a= \\
& \forall p .(\text { Alternative } p, \text { CharParser } p) \Rightarrow p a
\end{aligned}
$$

Under this definition, a rule is something that can be interpreted in any functor that supports the required primitives, e.g. $b s$ and $b s^{\prime}$ :

$$
\begin{aligned}
& b s, b s^{\prime}:: \text { FinalRule0 String } \\
& b s=(:) \circledast \text { token ' } \mathrm{b} \text { ' } \circledast \text { bs } \text { (1) pure " " } \\
& b s^{\prime}=\text { snoc } \$ b s^{\prime} \circledast \text { token ' } \mathrm{b} \text { ' (1) pure " " }
\end{aligned}
$$

The quantification over $p$ in FinalRule 0 rules out definitions like badBind. Since a term in the DSL has to support any functor $p$, it has no way of inspecting recursive references of type $p a$.

Writing algorithms in this final style is reminiscent of programming with Church encodings of data types. The polymorphism of FinalRule 0 is used by instantiating $p$ with a special-purpose interpretation functor carrying intermediate analysis results. In the instances of Alternative and CharParser for this functor, parsing primitives like pure, $\Phi$ and token are handled. The analysis function nullableF just unwraps the result from the functor:

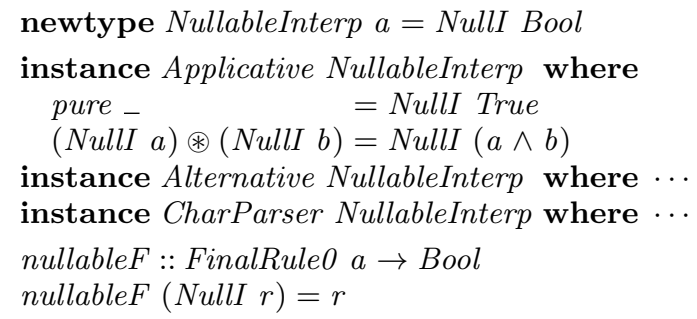

\subsection{Finally Recursive (third attempt)}

So how can we add a recursive binder to such a final DSL? The obvious solution mimics Carette et al.'s lambda construct:

$$
\begin{aligned}
& \text { class ApplicativeFix } 0 \text { where } \text { afix }_{0}::(p a \rightarrow p a) \rightarrow p a \\
& b s=a_{\text {fix }} \$ \lambda b s_{0} \rightarrow(:) \text { (token ' } \mathrm{b} \text { ' } \circledast b s_{0} \quad \text { (1) pure " " } \\
& b s^{\prime}=a_{i f x_{0}} \$ \lambda b s_{0}^{\prime} \rightarrow \text { snoc } \$ b s_{0}^{\prime} \circledast \text { token 'b' (1) pure " " }
\end{aligned}
$$

We can extend our nullableF analysis to support $a f i x_{0}$ :

$$
\begin{aligned}
& \text { instance ApplicativeFix } x_{0} \text { NullableInterp where } \\
& \text { afix } x_{0} p f=p f \text { (NullI False) }
\end{aligned}
$$

This instance passes NullI False to $p f$, specifying that recursive occurrences are assumed not to accept the empty string.

\subsection{Applicative problems}

Unfortunately, a problem with $a f i x_{0}$ remains in complex transformations like the left-recursion removal transform to be described in section 6.2 It will transform for example the left-recursive rule $b s^{\prime}$ into an equivalent, non-left-recursive rule: 


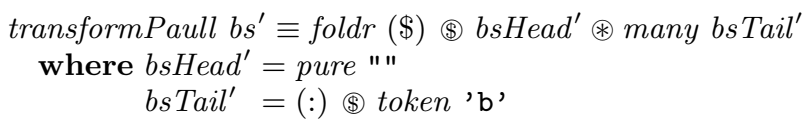

More details follow, but essentially $b s^{\prime}$ is split in two parts: $b s H e a d^{\prime}$ is what remains of the rule with leading self-references removed and $b s$ Tail $^{\prime}$ contains the parts of the rule that follow leading self-references. The transformed rule parses $b s \mathrm{Head}^{\prime}$ first and then iteratively parses $b s$ Tail $^{\prime}$ using many $:: p a \rightarrow p[a]$. The initial result of $b s H e a d^{\prime}$ has type String, and the parse results of bs Tail' ${ }^{\prime}$, typed String $\rightarrow$ String, are iteratively applied to it using foldr $(\$)$, to obtain the same parse results as before.

Actually performing this translation presents several challenges. For a rule like $b s^{\prime}$ of the form $a f i x_{0} b s f^{\prime}$, we have to derive rules bsHead ${ }^{\prime}$ and $b s$ Tail $^{\prime}$ from $b s f^{\prime}$. Distinguishing leading from nonleading recursive references is a first problem, but let us assume for now they are all leading (like in $b s^{\prime}$ ). It is then easy enough to find $b s H e a d^{\prime}$ as $b s f^{\prime}$ empty. Deriving $b s$ Tail $^{\prime}$ from $b s f^{\prime}$ is harder.

To derive $b s$ Tail $^{\prime}$ (of type $p$ (String $\rightarrow$ String)) from $b s f^{\prime}$ (of type $p$ String $\rightarrow p$ String), we can try to somehow turn $b s f^{\prime}$ into a value of type $p($ String $\rightarrow$ String $) \rightarrow p($ String $\rightarrow$ String $)$, i.e. make all rules produce a value that can depend on the String result of a previous match. The rules in $b s f^{\prime \prime}$ ignore this value, but we can now apply $b s f^{\prime \prime}$ to pure $i d$, i.e. instantiate leading selfreferences to a pure parser returning the previous parse result.

Generalising slightly, what we crucially need is a coapplicative operator coapp0 (note the symmetry with the type of $\circledast$ ):

$$
\text { coapp0 :: }(p a \rightarrow p b) \rightarrow p(a \rightarrow b)
$$

coapp0 should satisfy pf (pure $v) \equiv(\$ v)$ (\$) coapp0 pf for pf :: $p a \rightarrow p b$ and $v:: a$. However, it cannot be implemented ${ }^{4}$

We might say that the polymorphism of FinalRule0 has to be instantiated too early. If we could instantiate $p$ to $(p \circ((\rightarrow) a))^{5}$ then we could lift the Applicative and related instances to this composed functor, lift pure id to type $(p \circ((\rightarrow) a)) a$ and call $p f$ with this value to obtain a value of $(p \circ((\rightarrow) a)) b$ which is essentially $p(a \rightarrow b)$. However, even though we can instantiate a FinalRule 0 's $p$ parameter as we like, $(p \circ((\rightarrow) a))$ is not suited as the type $a$ becomes known only during the analysis of a term.

We note that coapp0's type is precisely that of Carette et al.'s Lambda constructor. So why can't we simply add a lambda constructor to our applicative DSL to solve our problem? Unfortunately, such a constructor is a bad idea for many DSLs, including our parsing example. Even for a simple recursive descent (RD) parse algorithm, this lambda operator cannot be handled:

$$
\begin{aligned}
& \text { lambdaRD }::((\text { String } \rightarrow[(\text { String }, a)]) \rightarrow \\
& \quad \text { String } \rightarrow[(\text { String }, a)]) \rightarrow \text { String } \rightarrow[(\text { String }, a \rightarrow b)] \\
& \text { lambdaRD pf } s=?
\end{aligned}
$$

\subsection{Rank-2 types to the rescue (our final proposal)}

The solution we propose is to change the type signature of $a f i x_{0}$ :

$$
\begin{gathered}
\text { class Applicative } p \Rightarrow \text { ApplicativeFix } p \text { where } \\
\text { afix }::(\forall \text { q Applicative } q \Rightarrow \\
(p \circ q) a \rightarrow(p \circ q) a) \rightarrow p a
\end{gathered}
$$

afix's type requires that the value of the recursive variable of $a$ fix's argument can be wrapped in an arbitrary Applicative functor $q$

\footnotetext{
${ }^{4}$ We ignore unsafe techniques like dynamic typing and partiality.

${ }^{5}(\rightarrow)$ is Haskell's curried arrow type constructor: $(\rightarrow) a b=a \rightarrow b$.
}

with the recursive definition's result value coming out in the same functor at the end.

This restriction is strong enough for the definition of coapp. We can instantiate the type constructor argument $q$ to the environment functor $((\rightarrow) a)$, and exploit its standard Applicative instance:

$$
\begin{gathered}
\text { coapp }:: \text { Applicative } p \Rightarrow(\forall q . \text { Applicative } q \Rightarrow \\
\quad(p \circ q) a \rightarrow(p \circ q) b) \rightarrow p(a \rightarrow b) \\
\text { coapp } p=\text { comp } \$ p \$ \text { liftInner } i d
\end{gathered}
$$

Our experience shows that afix's type is not too restrictive though: Applicative-style primitives on a functor $p$ can be lifted to $(p \circ q) a$ for any Applicative q, e.g. Alternative and CharParser 6

$$
\begin{gathered}
\text { instance }(\text { Alternative } p, \text { Applicative } q) \Rightarrow \\
\text { Alternative }(p \circ q) \text { where } \\
\text { empty }=\text { Comp empty } \\
v a \oplus v b=\text { Comp }(\text { comp va }(\text { D comp } v b) \\
\text { instance }(\text { CharParser } p, \text { Applicative } q) \Rightarrow \\
\text { CharParser }(p \circ q) \text { where } \\
\text { token }=\text { liftOuter } \cdot \text { token }
\end{gathered}
$$

We can adapt the previous examples $b s$ and $b s^{\prime}$ as follows.

$$
\begin{aligned}
& \text { type FinalRule } a=\forall p \text {. } \\
& \quad(\text { Alternative } p, \text { ApplicativeFix } p, \text { CharParser } p) \Rightarrow p a \\
& b s, b s^{\prime}:: \text { FinalRule String } \\
& b s=\text { afix } \$ \lambda s \rightarrow(:) \circledast \text { token ' } \mathrm{b} \text { ' } \circledast s \quad \text { (1) pure " " } \\
& b s^{\prime}=\text { afix } \$ \lambda s \rightarrow \text { snoc }(\$ s \circledast \text { token ' } \mathrm{b} \text { ' } \text { (1) pure " " }
\end{aligned}
$$

The additional power of $a f i x$ over $a f i x_{0}$ is not always needed. In such cases (like our nullable), the quantified functor $q$ can be instantiated to Identity:

$$
\begin{aligned}
& \text { runIdComp }:: \text { Functor } p \Rightarrow(p \circ \text { Identity }) a \rightarrow p a \\
& \text { runIdComp } p=\text { runIdentity } \$ \text { comp } p \\
& \text { wrapIdComp }:: \text { Applicative } p \Rightarrow(\forall q \text {. Applicative } q \Rightarrow \\
& \quad(p \circ q) a \rightarrow(p \circ q) \text { a) } \rightarrow p a \rightarrow p a \\
& \text { wrapIdComp } f=\text { runIdComp } \$ \text { f } \$ \text { liftOuter } s \\
& \text { instance ApplicativeFix NullableInterp where } \\
& \quad \text { afix } p f=\text { wrapIdComp pf (NullI False) }
\end{aligned}
$$

With firstSet similarly adapted, the results for $b s$ are unchanged: nullableF $b s \equiv$ True, firstSet $F$ bs $\equiv$ singleton ' $\mathrm{b}$ ', but left-recursion is now supported: nullableF $b s^{\prime} \equiv$ True and firstSetF $b s^{\prime} \equiv$ singleton 'b'.

\subsection{The meaning of afix's type}

Let us try and gain more insight into the meaning of afix's type:

$$
\begin{aligned}
& \text { afix }:: \forall p \text {. ApplicativeFix } p \Rightarrow \\
& \quad(\forall q . \text { Applicative } q \Rightarrow p(q a) \rightarrow p(q a)) \rightarrow p a
\end{aligned}
$$

A useful tool is Reynolds' notion of parametricity [32, 34]. Because we just want to gain insight, we freely use imprecise formulations and ignore intricacies like strictness [8], although we do believe the results could be made more exact if necessary.

Let us consider the type of afix's arguments:

$$
\forall \text { q. Applicative } q \Rightarrow(p \circ q) a \rightarrow(p \circ q) a
$$

We conjecture that the following free theorem holds for values $p f$ of this type:

\footnotetext{
${ }^{6}$ This instance of Alternative $(p \circ q)$ arbitrarily lifts $p$ 's Alternative instance and not $q$ 's, but in our case, $q$ 's special role warrants this.
} 
Theorem 3.1. For all $q_{1}, q_{2}$ Applicative, for $k: q_{1} a \rightarrow q_{2} a$ respecting the Applicative operations and $u::(p \circ q 1) a$,

$$
\text { withInner } k(p f u) \equiv p f(\text { withInner } k u) \text {. }
$$

This theorem states that for any $k$, applying withInner $k$ before or after $p f$ has the same effect. Consider the constant functor $K$ :

data $K a=K$

instance Applicative $K$ where $\left\{\right.$ pure $\left.{ }_{-}=K ;_{-} \circledast{ }_{-}=K\right\}$

With $k=\lambda_{-} \rightarrow K$, withInner $k$ maps values of $(p \circ q) a$ to $(p \circ K) a$, effectively erasing the value of the computation in $p$. Theorem 3.1 then implies that applying $p f$ to an argument $u$ and erasing the value of the result is equivalent to first erasing $u$ 's value and then applying $p f$ to it. This means that the above theorem states a familiar property, namely that $a f i x$ 's arguments $p f$ respect the separation between values and effects of applicative functors.

\section{An ApplicativeFix Axiom}

With the key points of our approach established, let us consider the properties a reasonable implementation of ApplicativeFix should satisfy. Many Haskell type classes are associated with such axioms that can sometimes even be linked to category theory or mathematics in general. We have not identified such a relation, but we do propose an axiom that should hold for $a f i x$.

\subsection{Fixpoint law}

The ApplicativeFix fixpoint law states that an implementation of afix should always deliver a fixpoint:

$$
\text { afix } p f \equiv \text { wrapIdComp pf } \$ \text { afix } p f
$$

Remember that denotationally, Haskell recursive definitions produce the least fixpoint of a function $p f$ [36]. This law states that afix must also return a fixpoint, but not necessarily the least. Note by the way that it also implies a behaviour on constant functions:

Corollary 4.1 (Constant preservation). If $x$ is not free in $g$, afix $(\lambda x \rightarrow$ liftOuter $g) \equiv g$.

\subsection{Interesting non-axioms}

There are two axioms that we want to explicitly not propose. We discuss them, nevertheless, as they shed more light on the meaning of our afix primitive.

Left shrinking considered harmful The first such property is an analogon of Erkök and Launchbury's Left Shrinking law for MonadFix [14] or the equivalent for Paterson's ArrowLoop [27]. For ApplicativeFix, one might expect the following left shrinking:

$$
\text { afix }(\lambda x \rightarrow \text { liftOuter } a \circledast f x)=a \circledast \text { afix } f
$$

with $x$ not free in $a$ and $a:: p(v \rightarrow v)$ for some $p$ and $v$.

Let us consider what this proposed axiom would mean for an example parser for an infinite number of $a$ s, defined as follows:

$$
a s=a f i x \$ \lambda s \rightarrow(:) \circledast \text { token ' } \mathrm{a} \text { ' } \circledast s
$$

Under the left-shrinking assumption, the above is equivalent to a single token parser followed by an infinite effect-free parser:

$$
\begin{aligned}
a s & =\text { afix } \$ \lambda a s^{\prime} \rightarrow(:) \circledast \text { token ' } \mathrm{a} \text { ' } \circledast a s^{\prime} \\
& =(:) \circledast \text { token ' } \mathrm{a} \text { ' } \circledast \text { afix } \$ \lambda a s^{\prime} \rightarrow a s^{\prime}
\end{aligned}
$$

However, this new expression is not at all equivalent to the original. The left shrinking property states that computations not depending on recursive occurrences can be lifted out of the recursive equation, their effects no longer taking part in the recursion. As such, the axiom is defining for the value recursion that Erkök and Launchbury model and rules out the effectful recursion we aim for.

Fixing what is pure? A second property that not all reasonable instances of ApplicativeFix satisfy, prescribes a form of fixpoints for recursive definitions that do not add effects:

$$
\text { afix }(\text { fmap } h) \equiv \operatorname{pure}(\text { fix } h)
$$

for any $h$ typed $a \rightarrow a$. The right hand side is a fixpoint of fmap $h$, but not necessarily the one we want. In an Alternative functor $p$, empty is also a fixpoint and it is in fact the natural one for most examples in this text. For instance, in a parser DSL, the lefthand side models a non-terminal $X$ with a single production rule $X \rightarrow X$, equivalent in language theory to an empty rule.

\section{Making afix practical}

We turn our attention to some tools that make it practical to implement and work with DSLs using $a$ fix.

\subsection{Some Tools}

First, unlike alternative solutions [2, 11], afix supports higherorder combinators without primitive support. These are observably recursive analogues of the standard many and some combinators:

$$
\begin{aligned}
& \text { manyAF, someAF :: (Alternative } p, \text { ApplicativeFix } p) \Rightarrow \\
& p a \rightarrow p[a] \\
& \text { someAF } f=(:) \circledast f \circledast \text { manyAF } f \\
& \text { many } A F f=\text { afix } \$ \lambda s \rightarrow(:) \circledast \text { liftOuter } f \circledast s \oplus \text { pure [] }
\end{aligned}
$$

Note that many $A F$ and someAF's types only differ from their standard analogues in the ApplicativeFix constraint.

A standard function afixInf implements afix by going back to Haskell's unobservable recursion. This sometimes makes sense, for example when interfacing with existing libraries that are designed to work with Haskell's implicit recursion. We show an instance for uu-parsinglib's parser representation. Also useful is afixKill, which replaces recursive occurrences with a failing empty rule.

$$
\begin{aligned}
& \text { afixInf :: Applicative } p \Rightarrow(\forall q \text {. Applicative } q \Rightarrow \\
& (p \circ q) a \rightarrow(p \circ q) a) \rightarrow p a \\
& \text { afixInf } f=\text { fix } \$ \text { wrapIdComp } f \\
& \text { instance ApplicativeFix ( } P \text { st) where } \text { afix }=\text { afixInf } \\
& \text { afixKill :: Alternative } p \Rightarrow(\forall q \text {. Applicative } q \Rightarrow \\
& (p \circ q) a \rightarrow(p \circ q) a) \rightarrow p a \\
& \text { afixKill } f=\text { runIdComp } \$ f \text { empty }
\end{aligned}
$$

\subsection{Arity-Genericity}

With afix modelling effectful recursion on one variable, the next question is what to do about mutual recursion. Instantiating $a f i x$ at type $(\forall b$. ApplicativeFix $b \Rightarrow(f \circ b)(a 1, a 2) \rightarrow(f \circ$ b) $(a 1, a 2)) \rightarrow f(a 1, a 2)$ is not a solution because the type $f(a 1, a 2)$ does not allow both recurrands to produce different effects. What we would like is a primitive of the following type: 


$$
\begin{aligned}
& \text { assocComp }_{1}:: \text { Applicative } \Rightarrow \\
& \quad\left((p \circ q 1) \circ q^{2}\right) a \rightarrow\left(p \circ\left(q 1 \circ q^{2}\right)\right) a \\
& \text { assocComp }_{2}:: \text { Applicative } p \Rightarrow \\
& \quad\left(p \circ\left(q 1 \circ q^{2}\right)\right) a \rightarrow\left((p \circ q 1) \circ q^{2}\right) a \\
& \text { liftComposed }::(\text { Applicative } p, \text { Applicative q2) } \Rightarrow \\
& \quad(p \circ q 1) a \rightarrow\left(p \circ\left(q^{2} \circ q 1\right)\right) a
\end{aligned}
$$

Figure 1. Utility functions for working with functors $(p \circ q)$ (implementations omitted).

$$
\begin{aligned}
& \text { afix }_{2}:: \text { ApplicativeFix } p \Rightarrow(\forall q . \text { Applicative } q \Rightarrow \\
& \quad\left((p \circ q) a_{1} \rightarrow(p \circ q) a_{2} \rightarrow(p \circ q) a_{1},\right. \\
& \left.\left.\quad(p \circ q) a_{1} \rightarrow(p \circ q) a_{2} \rightarrow(p \circ q) a_{2}\right)\right) \rightarrow\left(\begin{array}{lll}
p & a_{1}, p & a_{2}
\end{array}\right)
\end{aligned}
$$

This is not an instance of afix's type, but we can construct such a primitive from it, taking inspiration from Bekić's theorem [36]. This theorem relates mutually recursive bindings to repeated applications of the fix operator. We imitate the form for afix, exploiting the polymorphism of $a f i x_{2}$ 's argument and juggling the $(\cdot \circ \cdot)$ type constructor in the types (using utility functions from Figure 1 ).

$$
\begin{gathered}
a f i x_{2} p f=\left(f p_{1}, f p_{2}\right) \text { where } \\
f p_{1}=a f i x \$ \lambda f p_{1}^{\prime} \rightarrow \\
\text { let } f p_{2}^{\prime}=\text { Comp } \$ \text { afix } \$ \lambda f p_{2}^{\prime \prime} \rightarrow \text { comp } \$ \\
\text { assocComp } p_{2} \$ \text { snd pf }\left(\text { liftComposed } f p_{1}^{\prime}\right) \\
\quad\left(\text { assocComp } p_{1} \$ \text { Comp } f p_{2}^{\prime \prime}\right) \\
\text { in } f s t p f f p_{1}^{\prime} f p_{2}^{\prime} \\
f p_{2}=\ldots \text { (analogous) }
\end{gathered}
$$

This definition is obscured by technicalities. In the first call to afix, the recursive argument $f p_{1}^{\prime}$ is of type $(p \circ q 1) a_{1}$ for an arbitrary $q 1$. However, in the second call to $a f i x, f p_{2}^{\prime \prime}$ is of type ( $\left.p \circ q^{2}\right)\left(\begin{array}{ll}q 1 & a_{2}\end{array}\right)$ for an arbitrary $q 2$. The trick is then to apply $p f$ with its type parameter $q$ instantiated to $(q 2 \circ q 1)$ and exploit associativity of the $(\cdot \circ \cdot)$ type constructor to fit everything together.

Now, we can do this for any arity, but this is not very effective: we can only hope to implement a finite number of $a f i x_{i}$ s and additionally, the size of the definition of $a f i x_{i}$ increases exponentially with $i$. Luckily, GHC's type system allows us to do better.

Omitting details for space reasons, we have used techniques described by McBride $[22]^{7}$ to develop an arity-generic or polyvariadic version of $a$ fix. With encoding artefacts removed and using ellipses in a not fully formal notation for type lists, this is its type:

$$
\begin{aligned}
& \text { nafix }:: \forall(p:: * \rightarrow *)\left(\left[t_{1} \cdots t_{n}\right]::[*]\right) \text {. ApplicativeFix } p \Rightarrow \\
& \quad((\forall q: * \rightarrow * \text {.Applicative } q \Rightarrow \\
& \left.\quad(p \circ q) t_{1} \rightarrow \cdots \rightarrow(p \circ q) t_{n} \rightarrow(p \circ q) t_{1}\right), \cdots, \\
& \quad(\forall q: * \rightarrow * . \text { Applicative } q \Rightarrow \\
& \left.\left.\quad(p \circ q) t_{1} \rightarrow \cdots \rightarrow(p \circ q) t_{n} \rightarrow(p \circ q) t_{n}\right)\right) \rightarrow \\
& \quad\left(p t_{1}, \ldots, p t_{n}\right)
\end{aligned}
$$

This arity-generic version of afix gives us effectful mutual recursion at any arity without primitives beyond $a f i x$. The implementation of nafix can be found in our GHC branch (see Section 5.4).

\subsection{Syntactic sugar}

By writing recursive definitions in terms of this new fixpoint primitive, we lose some of the elegance of Haskell's standard let bindings. But because our ApplicativeFix and afix are a general tool

\footnotetext{
${ }^{7}$ We use type families instead of multi-parameter type classes though.
}

$$
\begin{gathered}
Q ; \Gamma \vdash p: * \rightarrow * \quad Q \Vdash \text { ApplicativeFix } p \quad \mathrm{q} \text { fresh } \\
\forall i=1 . . n, Q \wedge \text { Applicative } q ; \\
\Gamma, q: * \rightarrow *,\left\{x_{j}:(p \circ q) t_{j}\right\}_{j=1 . . n} \vdash e_{i}:(p \circ q) t_{i} \\
Q ; \Gamma,\left\{x_{i}: p t_{i}\right\}_{i=1 . . n} \vdash e: T \\
Q ; \Gamma, \vdash \text { alet }\left\{x_{i}=e_{i}\right\}_{i=1 . . n} \text { in } e: T
\end{gathered}
$$

Figure 2. Typing alet bindings

for Applicative functors requiring observable effectful recursion, a shorthand notation to recover this elegance makes sense. Since we work in an applicative style, a syntax that resembles standard let bindings is natural: we propose the alet-notation:

alet $b s=$ pure " " $($ (:) $($ token ' $\mathrm{b}$ ' $\circledast b s$

Note the use of a top-level alet. We allow mutual recursion, like in the following simple expression parser ${ }^{8}$

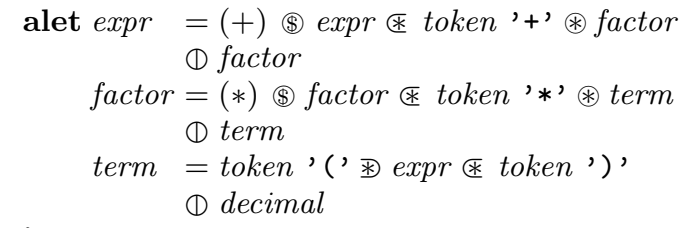

in $\operatorname{expr}$

Syntactic and scoping rules for alet are analogous to normal let bindings. Its typing rule is presented in Figure 2 , using notation from Vytiniotis et al. [33]. Specifically, their typing judgement $Q ; \Gamma \vdash e: \tau$, where $Q$ is a set of constraints, $\Gamma$ is a typing environment, $e$ an expression and $\tau$ a type. We write some kind annotations, typing context entries for type variables and explicit type applications of polymorphic values for clarity. The typing rule for alet is not standard, but it reflects the requirements of our $a f i x$ and nafix primitives: the definitions of bound variables $x_{i}$ are typechecked against type $(p \circ b) t_{i}$ where $b$ is a fresh Applicative functor, and all $x_{j}$ are bound at type $(p \circ b) t_{j}$. The body of the alet construct is type-checked with all $x_{i}$ bound at type $p t_{i}$.

With this typing rule, Figure 3 defines a type- and syntax-directed desugaring $\mathcal{A}$, transforming alet to regular Haskell. Essentially, all recursively bound variables $x_{i}$ are converted into open recursive functions $x_{i}^{\sharp}$. We generate a call to the $n$-ary nafix primitive and project out $\hat{x}_{i}$ from the resulting n-ary tuple. Finally, we replace occurrences of $x_{i}$ in the body with $\hat{x}_{i}$. Type soundness of the translation follows easily from type checking the right-hand side of the translation $\mathcal{B}$ against the type of the original alet construct.

Theorem 5.1 ( $\mathcal{A}$ is type-preserving).

$$
Q ; \Gamma \vdash e: T \Rightarrow Q ; \Gamma \vdash \mathcal{A}\langle\Gamma\rangle(e): T \text {. }
$$

We don't formalise top-level alets, a declaration form of alets.

\subsection{Implementation}

We have extended GHC to parse, type-check and desugar alet expressions. We perform the translation in Fig. 3 during the desugaring phase, which translates scope- and type-checked Haskell code into GHC's explicitly typed core language (a variant of System $F_{\omega}$ extended with features like equality coercions [30]). We currently support alet expressions as defined in this text. Still missing is support for top-level alets and type signatures for alet bindings

\footnotetext{
${ }^{8}$ Note: we use shorthands $\circledast$ and $\circledast$ that behave like $\circledast$ but ignore the result of their second resp. first argument, e.g. $v \circledast w=$ const $\$ v \circledast w$.
} 


$$
\begin{aligned}
& \mathcal{A}\langle Q ; \Gamma\rangle\left(\text { alet }\left\{x_{i}=e_{i}\right\}_{i=1 . . n} \text { in } e\right)=\mathcal{B}\left\langle\left\{t_{i}\right\}_{i=1 . . n}\right\rangle\left(\text { alet }\left\{x_{i}=e_{i}\right\}_{i=1 . . n} \text { in } e\right) \\
& \text { with }\left\{\begin{array}{l}
Q ; \Gamma \vdash p: * \rightarrow *, \quad Q \Vdash \text { ApplicativeFix } p \\
\forall i Q \wedge \text { Applicative } b ; \Gamma, b: * \rightarrow *,\left\{x_{j}:(p \circ b) t_{j}\right\}_{j=1 . . n} \vdash e_{i}:(p \circ b) t_{i}
\end{array}\right. \\
& \mathcal{A}\langle Q ; \Gamma\rangle(e) \quad=\ldots \quad \text { (compositionally apply } \mathcal{A}\langle Q ; \Gamma\rangle \text { on components of } e \text { ) } \\
& \mathcal{B}\left\langle\left\{t_{i}\right\}_{i=1 . . n}\right\rangle\left(\text { alet }\left\{x_{i}=e_{i}\right\}_{i=1 . . n} \text { in } e\right)=\text { let } \quad\left\{x_{i}^{\sharp}=\lambda x_{1}^{\prime} \cdots x_{n}^{\prime} \rightarrow\left[x_{j}^{\prime} / x_{j}\right]_{j=1 . . n} e_{i}\right\}_{i=1 . . n} \\
& f=n a f i x @\left[t_{1} . . t_{n}\right]\left(x_{1}^{\sharp}, \cdots, x_{n}^{\sharp}\right) \\
& \left\{\hat{x}_{i}=\pi_{i} @\left[\left(p t_{1}\right) \cdots\left(p t_{n}\right)\right] f\right\}_{i=1 . . n} \\
& \text { in }\left[\hat{x}_{i} / x_{i}\right]_{i=1 . . n} e
\end{aligned}
$$

Figure 3. The type-directed translation of alet bindings. We write some explicit type applications for clarity.

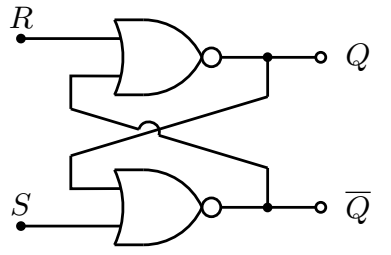

Figure 4. An SR-latch, using a pair of cross-coupled NOR gates.

(but type annotations in the bodies can be used instead). The examples in this text without top-level alets are supported. The code is available on GitHub with instructions for building ${ }^{9}$ and pointers for trying out examples and navigating the code.

\section{Examples}

\subsection{Functional Reactive Programming}

A domain where our techniques are useful is functional reactive programming (FRP), a declarative programming model that describes a system in terms of time-varying functions instead of mutable state with applications in robotics [28], animation [13] and graphical user interfaces [19].

A simple FRP model may consider behaviours as functions of time, making them an instance of the environment functor [23]:

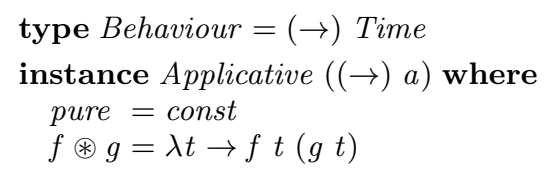

FRP provides a form of state through the delaying of behaviours. For discrete time models, the behaviour delay $v$ bhv produces value $v$ first and the delayed values of $b h v$ next. We assume functions prevT :: Time $\rightarrow$ Time and initT :: Time.

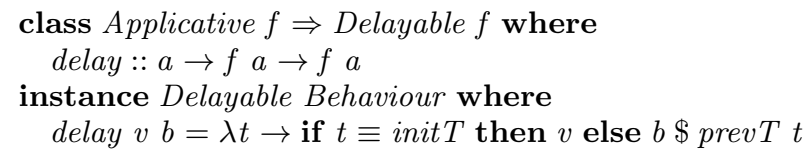

\subsubsection{Modelling electronic circuits with flip-flops}

Let us use FRP to model event networks and, in particular, electronic circuits. Figure 4 shows an SR-latch, a simple flip-flop cir-

\footnotetext{
http://github.com/ilyasergey/GHC-XAppFix/wiki
}

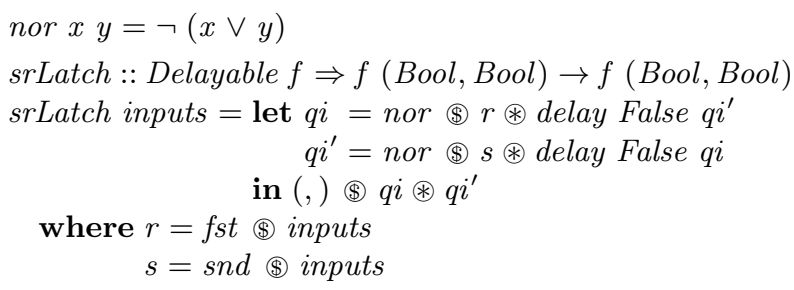

Figure 5. An FRP implementation of an SR latch's without alet.

cuit with two stable states, sometimes used to store information. The circuit has two input wires: $R$ and $S$, and two output wires: $Q$ and $\bar{Q}$ (opposite in correct states). If $S$ and $R$ are high, they set resp. reset the state of the circuit (the value of $Q$ ).

Figure 5 shows an FRP model of the SR-latch over an arbitrary Applicative functor with a Delayable instance, reacting to events of type (Bool, Bool) (the values applied to the input wires). The latch's recursion is modelled in the standard way, and there is no problem to simulate the circuit:

$$
\text { sampleInput }=\text { delay }(\text { True, False) } \$ \text { const }(\text { False, False })
$$

The simulation shows that our sample input correctly initialised the circuit: map (srLatch sampleInput) [initT...] gives us $[($ False, True $),($ False, True $), \ldots]$.

\subsubsection{Delayable applicative functors}

In Figure 5 the recursive calls to $q i$ and $q i^{\prime}$ are guarded by delays, essential for the proper functioning of the example. If we remove the delays, our example no longer terminates. For more complex examples, such errors can be less obvious and lead to bugs.

Our approach can already do better. If we model the recursion using ApplicativeFix, we can scan for erroneous loops upfront and report errors early on. We just need to lift the Delayable class to composed functors (omitted) and change srLatch to use alet. We use liftOuter to lift $a$ and $b$ into the composed functor:

$$
\begin{gathered}
\text { srLatch2 }::(\text { ApplicativeFix } f, \text { Delayable } f) \Rightarrow \\
f(\text { Bool, Bool }) \rightarrow f(\text { Bool, Bool }) \\
\text { srLatch2 inputs }= \\
\text { alet } q i=\text { nor }\left(\text { liftOuter } r \circledast \text { delay False } q i^{\prime}\right. \\
q i^{\prime}=\text { nor } \$ \text { liftOuter } s \circledast \text { delay False } q i \\
\text { in }(,)(\$) \text { li } q i^{\prime} \\
\text { where } \ldots \text {-. see Figure } 5
\end{gathered}
$$

In a final style, we can analyse this definition with a custom functor: Test Valid $a$ ignores its parameter $a$, but keeps track of the valid- 
ity of a circuit and its current minimum delay. The Applicative and Delayable instances initialise and combine the validity and minimum delay values in the obvious way.

$$
\begin{aligned}
& \text { data ValidInterp } a=V I\{\text { is Valid }:: \text { Bool, viDelay }:: \text { Int }\} \\
& \text { instance Applicative ValidInterp where } \\
& \quad=V I \text { True } 0 \\
& \text { pure }- \text { VI } d a \circledast V I v b d b=V I(v a \wedge v b)(\text { min } d a d b) \\
& \text { instance Delayable ValidInterp where } \\
& \quad \text { delay }-(V I s d)=V I s(d+1)
\end{aligned}
$$

In the ApplicativeFix instance, we test if the recursive function properly introduces a delay: we pass it a recursive occurrence with a delay of -1 . If what comes back doesn't have an increased delay, the circuit is invalid:

$$
\begin{aligned}
& \text { instance ApplicativeFix ValidInterp where } \\
& \text { afix } f=\text { case wrapIdComp } f \text { VI True }(-1) \text { of } \\
& \text { VI } t d \mid d<0 \quad \rightarrow \text { VI False } d \\
& V I t d \mid \text { otherwise } \rightarrow \text { VI } t d
\end{aligned}
$$

Reassuringly, is Valid (srLatch2 (VI True 0)) is True.

\subsection{Left-recursion removal}

The largest example in this paper is the previously mentioned algorithm that transforms left-recursive parsers into an equivalent nonleft-recursive form. It is inspired by a previous implementation of a uniform version of the Paull transformation [17. p. 304] in the grammar-combinators library [11]. This is a complex transformation and we think it shows other transformations like the ones needed for parsing with derivatives [24] are possible as well.

In section 3.3 , we discussed how transformPaull transforms the example left-recursive rule $b s^{\prime}$ into a non-left-recursive equivalent:

$$
\begin{aligned}
& \text { transformPaull bs }{ }^{\prime} \equiv \\
& \text { foldr }(\$)\left(\text { bsHead }^{\prime} \circledast\right. \text { many bsTail' where } \\
& \text { bsHead' }=\text { pure " " } \\
& \text { bsTail' }=(:) \circledast \text { token ' } \mathrm{b} \text { ' }
\end{aligned}
$$

The core of the transformation is in the implementation of $a$ fix, where we transform a parser like $b s^{\prime}=a f i x b s f^{\prime}$ to the above. The difficult part is deriving bsHead ${ }^{\prime}$ and $b s$ Tail $^{\prime}$ from $p f$ and we have already discussed some of the techniques for doing this in section 3 To derive the first, we pass $p f$ a recursive occurrence that behaves differently in different positions: it always fails in a head position and behaves as an actual recursive occurrence in tail positions. In a similar but more complicated way, we construct bs Tail $^{\prime}$, but not without the coapp function from section 3.4

We model different positions for a parsing rule as three contexts: PurePos (a position where a parser must not consume any input), HeadPos (the left-most position of a non-terminal definition where a parser must consume input) and TailPos (a position where input has already been consumed). The HeadPos context can additionally specify that primitive parsers in this position are to be either killed (replaced with empty) or left unmodified:

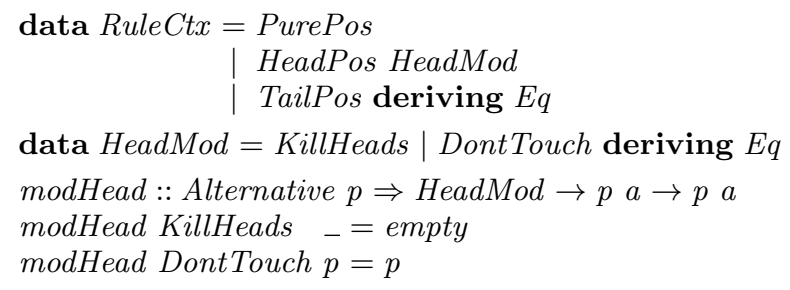

For an underlying parser functor $p$, we define a type of transformable parsers that support these three contexts. This type of transformable rules plays the role of the interpretation functor that we instantiate the polymorphism of the rules with. We define the algorithm by providing instances for the relevant type classes Applicative, Alternative, CharParser and ApplicativeFix.

data PaullT $p$ a = PaullT $\{$ paullT $::$ RuleCtx $\rightarrow p$ a $\}$

The Alternative instance just lifts $p$ 's operations in all contexts:

instance Alternative $p \Rightarrow$ Alternative (PaullT $p$ ) where empty $=$ PaullT $\$$ const empty

$a \oplus b=$ PaullT $\$ \lambda$ ctx $\rightarrow$ paullT a ctx (1) paullT $b$ ctx

In the Applicative instance, we define the behaviour of the parsers in the three contexts. The rule for sequencing is the most complex, because care has to be taken to correctly define how the two sequenced parts can each be in the left-most position of a rule:

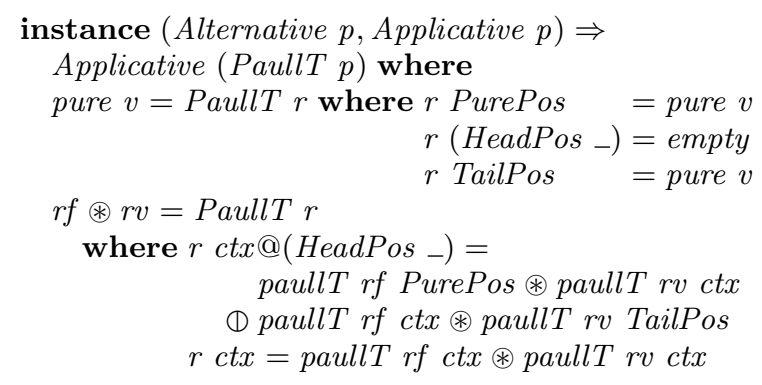

The CharParser instance is self-explanatory:

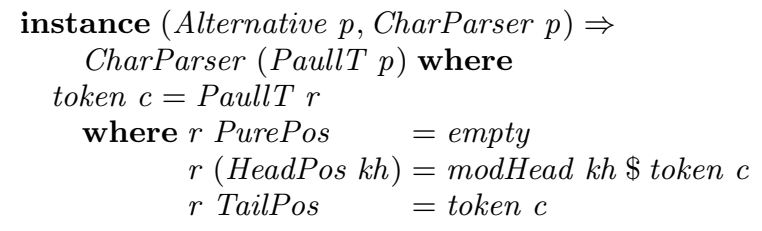

Finally, the ApplicativeFix instance handles recursive references:

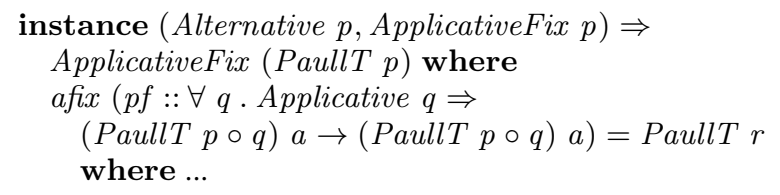

In a pure context, we just kill recursive occurrences:

$$
r \text { PurePos }=\text { paullT (afixKill pf) PurePos }
$$

In other contexts, we implement the transformation rule described above (taking into account the head modification in a head context). We use an omitted function manyComp: a version of manyAF from Section 5.1. lifted to composed functor $(p \circ q)$ :

$$
\begin{aligned}
& r(\text { HeadPos } h m)=r N P h m \\
& r \text { TailPos }=r N P \text { DontTouch } \\
& r N P:: \text { HeadMod } \rightarrow p \text { a } \\
& r N P h m=\text { afix } \$ \lambda \text { self } \rightarrow \\
& \quad \text { foldr }(\$) \text { (\$Start } h m \text { self } \circledast \text { manyComp }(r \text { Deriv self })
\end{aligned}
$$

rStart and rDeriv play the role of bsHead' and bs Tail' in our earlier explanation. We define rStart by calling the function $p f$ with a recursive occurrence that behaves as empty in pure and head contexts, and as an actual recursive reference elsewhere:

$r$ Start :: Applicative $q \Rightarrow$ HeadMod $\rightarrow(p \circ q) a \rightarrow(p \circ q) a$ rStart $h m$ self $=$ 


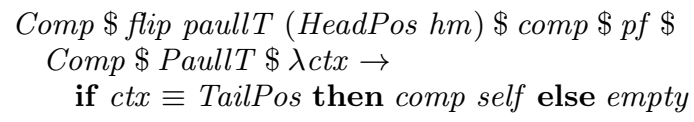

Finally, as discussed in Section 3.4 r Deriv is a prime example for the need of the coapp function described. It produces a parser with result type $a \rightarrow a$ to be used in the definition of $r N P$ above. In addition to the recursive occurrence self from the call to afix, coapp gives us a placeholder prev to substitute for left-recursive occurrences. Using our RuleCtxs, we call $f$ with a recursive reference behaving as prev in head positions and as self elsewhere:

$$
\begin{aligned}
& r \text { Deriv }:: \text { Applicative } q \Rightarrow(p \circ q) a \rightarrow(p \circ q)(a \rightarrow a) \\
& r \text { Deriv self }=\text { coapp } \$ r \text { Deriv' self } \\
& \text { rDeriv' }:: \forall q \text { q2 . (Applicative } q \text {, Applicative q2) } \Rightarrow \\
& (p \circ q) a \rightarrow((p \circ q) \circ q 2) a \rightarrow\left((p \circ q) \circ q^{2}\right) a \\
& r \text { Deriv' self prev }= \\
& \text { assocComp }_{2} \$ \text { Comp } \$ \\
& \text { flip paullT (HeadPos KillHeads) \$ comp \$ } \\
& \text { pf } \$ \text { Comp } \$ \text { PaullT rself where } \\
& \text { rself :: RuleCtx } \rightarrow p\left(q \circ q^{2}\right) a \\
& \text { rself PurePos = empty } \\
& \text { rself HeadPos }\{\}=\text { comp } \$ \text { assocComp }_{1} \text { prev } \\
& \text { rself TailPos = liftOuter (\$) comp self }
\end{aligned}
$$

With this machinery, the function transformPaull applies the leftrecursion removal transformation to a parser:

$$
\begin{array}{r}
\text { transformPaull }:: \text { Alternative } q \Rightarrow \text { PaullT } q a \rightarrow q a \\
\text { transformPaull } p=\text { paullT p PurePos } \\
\text { (1) paullT } p \text { (HeadPos DontTouch) }
\end{array}
$$

Omitting some uninteresting glue code to the standard Applicative parsing library uu-parsinglib, we can now apply its standard error-correcting parsing algorithm to our transformed and no longer left-recursive parser expr from Section 5.3

$$
\begin{aligned}
& \text { exprParse }:: \text { String } \rightarrow \text { Int } \\
& \text { exprParse }=\text { parseUU } \$ \text { transformPaull expr } \\
& \text { testParse }=\text { exprParse } 1+7 * 3+(8 * 1+2 * 6) "
\end{aligned}
$$

\section{Related Work}

Applicative functors For background on applicative functors, we refer to McBride and Paterson [23]. Note that their bracket notation (translating $\llbracket f u_{1} \ldots u_{n} \rrbracket$ to pure $f\left(u_{1} \circledast \ldots \circledast u_{n}\right.$ ) is orthogonal to our alet syntax and would fit well in alet right-hand sides. Lindley et al. clarify the relation between Applicative functors, Arrows and Monads [20]. Applicative parser combinators were popularised by Swierstra and colleagues [1, 31], who have shown them better suited for analysis and optimisation. A better handling of recursion is still a missing piece of the puzzle, and has been a motivation for work on observable recursion (see below).

HOAS representations There is a wide variety of research on HOAS representations and how to prevent the problem discussed in section 3 We have already discussed Carette et al. [5]'s finally tagless style, built on the work by Washburn and Weirich [35]. Chlipala has proposed Parametric HOAS, also exploiting parametricity in a better HOAS encoding [6]. Contrary to Carette et al., this results in an initial encoding instead of a final one. PHOAS has been used by Oliveira and Cook to obtain observable monomorphic recursion [25] in graph structures. In follow-up work developed in parallel with ours, Oliveira and Löh extend this to typed DSLs with mutually recursive bindings, leading to a solution with many similarities to ours [26]. In addition to observable recursion, they also provide observable sharing, which we have not considered. Their model of mutually recursive binders uses techniques similar to those in section 5.2 but they keep mutual bindings as primitive instead of reducing them to sequences of simple binders like us. For achieving a usable end-user syntax, they demonstrate the use of the impure data-reify library, while we propose the alet syntax.

We have experimented with using an initial encoding based on PHOAS instead of our finally tagless encoding. This involves a version of our Rule data type parameterised by a type constructor $v$ that represents variables of a certain type. We also add an additional Var constructor and a different recursion primitive Fix:

$$
\begin{aligned}
& \text { data PRule } v \text { a where } \\
& \text { Var }:: v a \rightarrow \text { PRule } v a \\
& \text { Fix }::(v a \rightarrow \text { PRule } v a) \rightarrow \text { PRule } v a \\
& \text { Seq }:: \text { PRule } v(a \rightarrow b) \rightarrow \text { PRule } v a \rightarrow \text { PRule } v b \\
& \quad \ldots \\
& \text { type Rule } a=\forall v \cdot \text { PRule } v a
\end{aligned}
$$

Parametricity of bindings is enforced by requiring the terms to support any variable type constructor $v$. However, this representation also suffers from the problem discussed in section 3.2 and does not permit the definition of an analogon of coapp. As in section 3.4 this can be solved by changing Fix's type to the rank-2 type

$$
\begin{aligned}
& \text { Fix }::(\forall w \text {. Applicative } w \Rightarrow \\
& \quad v:<: w \rightarrow w a \rightarrow \text { PRule } w a) \rightarrow \text { PRule } v a
\end{aligned}
$$

$v:<: w$ is a synonym for $\forall a \cdot v a \rightarrow w a$, i.e. an embedding of variables $v a$ into a wider set $w a$. We have not found reasons to prefer this encoding over ours, although some people may prefer an initial encoding over a final one. This encoding may translate better to predicative languages like Agda.

Observable recursion through fixpoint primitives We have already seen Erkök and Launchbury's MonadFix type class and fixpoint primitive $m f i x$, typed MonadFix $m \Rightarrow(a \rightarrow m a) \rightarrow$ $m a$ [14 15]. They focus on value recursion; mfix's type and its axioms (see Section 4.2) are not suited for effectful recursion. Erkök and Launchbury extend Haskell's do-notation with recursive value bindings that are desugared into applications of $m f i x$.

Hughes proposed the use of Arrows in functional languages [18] as a generalisation of monads, similar to applicative functors. In a paper proposing a do-notation for arrows, Paterson also proposed ArrowLoop: a type class modelling value recursion in arrows [27]. Similar to MonadFix, the ArrowLoop axioms prescribe a valuerecursion semantics and do not allow effectful recursion.

Observable recursion through typed references Both Baars and Swierstra [2, 3] and Devriese and Piessens [10, 11, 12] each define observably recursive encodings of grammars based on a well-typed representation of references. Baars and Swierstra employ a form of de Bruijn-indices into a type-level encoding of a type environment and Devriese and Piessens require the user to define an encoding of the grammar's non-terminals at both type and value level. Baars and Swierstra implement a fixpoint primitive and propose a general form of syntax macros for Haskell to recover a nice syntax for their grammar definitions. Brink et al. [4] demonstrate a deeper encoding of parsers than ours, representing grammars as a set of production rules parameterised by a set of non-terminals in the dependentlytyped language Agda. Compared to these approaches, our fixpoint primitive is more powerful. For example, we support higher order 
recursive operators like many $A F$ from Section 5.1 without hardcoded support in the parsing library.

Dependently typed recursion In an unpublished draft, Danielsson and Norell [9] show how left-recursive parsers can be excluded in a dependently typed parser combinator DSL. They restrict the recursion in the DSL while we support other interpretations of the recursion that is there. Danielsson [7] uses mixed induction-coinduction to define parser combinators that support left-recursion, but (impressively) remain provably total and correct. Danielsson's technique does not seem to allow the choice of non-standard fixpoints in the same way as we do. For the following parser test

$$
\begin{aligned}
& \text { test }: C o \mathbb{N} \rightarrow P \text { false } \\
& \text { test zero }=\text { sat }(\stackrel{?}{=} \text { ' } \mathrm{a} \text { ' }) \\
& \text { test }(\text { suc } n)=\sharp \text { test }_{2}(\mathrm{~b} n) \cdot \sharp ?(\text { sat }(\stackrel{\text { ? }}{=} \text { ' } \mathrm{b} \text { ' }))
\end{aligned}
$$

we suspect test $\infty$ is indistinguishable from finite test $n$ making first set calculation and other algorithms hopeless.

\section{Conclusion}

We have shown that effectful recursion is an important problem in several domains. We propose the class ApplicativeFix of Applicative functors with a recursion primitive afix. It uses a finally tagless HOAS encoding of a recursive binder $\mu$, adapted to Applicative functors with a rank-2 type that allows to exploit the Applicative values-effects separation. We use generic programming techniques to derive mutual recursion primitives and propose the alet construct as a shallow form of syntactic sugar for afix with an implementation in GHC. We show that our approach is useful for (at least) two domains: parsing and functional reactive programming. Our approach supports higher-order operators like many $A F$ without ad hoc support in the DSL encoding.

\section{Acknowledgements}

This research is partially funded by the Research Foundation - Flanders (FWO), and by the Research Fund KU Leuven. Dominique Devriese holds a Ph.D. fellowship of the Research Foundation Flanders (FWO). The authors thank Bruno Oliveira and Andres Löh for an interesting discussion about their related work.

\section{References}

[1] A. I. Baars, A. Löh, and S. D. Swierstra. Parsing permutation phrases. J. Funct. Program., 14(6):635-646, 2004.

[2] A. I. Baars and S. D. Swierstra. Type-safe, self inspecting code. In Haskell, pages 69-79, 2004.

[3] A. I. Baars, S. D. Swierstra, and M. Viera. Typed transformations of typed abstract syntax: The left corner transform. In LDTA, pages 18-33, 2009.

[4] K. Brink, S. Holdermans, and A. Löh. Dependently typed grammars. In $M P C, 2010$.

[5] J. Carette, O. Kiselyov, and C.-c. Shan. Finally tagless, partially evaluated: Tagless staged interpreters for simpler typed languages. J. Funct. Program., 19(05):509-543, 2009.

[6] A. Chlipala. Parametric higher-order abstract syntax for mechanized semantics. In ICFP, pages 143-156, 2008.

[7] N. A. Danielsson. Total parser combinators. In ICFP, pages 285-296, 2010.

[8] N. A. Danielsson, J. Hughes, P. Jansson, and J. Gibbons. Fast and loose reasoning is morally correct. In POPL, pages 206-217, 2006.
[9] N. A. Danielsson and U. Norell. Structurally recursive descent parsing. Draft, 2008.

[10] D. Devriese and F. Piessens. Explicitly recursive grammar combinators - Implemention of some grammar algorithms. Technical Report CW594, KULeuven CS, 2010.

[11] D. Devriese and F. Piessens. Explicitly recursive grammar combinators. In PADL, pages 84-98. Springer, 2011.

[12] D. Devriese and F. Piessens. Finally tagless observable recursion for an abstract grammar model. Journal of Functional Programming, 22(06):757-796, 2012.

[13] C. Elliott and P. Hudak. Functional reactive animation. In ICFP, pages 263-273, 1997.

[14] L. Erkök and J. Launchbury. Recursive Monadic Bindings. In ICFP, pages 174-185, 2000.

[15] L. Erkök and J. Launchbury. A recursive do for Haskell. In Haskell, pages 29-37, 2002.

[16] R. Frost, R. Hafiz, and P. Callaghan. Parser combinators for ambiguous left-recursive grammars. In PADL, 2008.

[17] J. E. Hopcroft, R. Motwani, and J. D. Ullman. Introduction to automata theory, languages, and computation. Addison-Wesley, 2003.

[18] J. Hughes. Generalising monads to arrows. Sci. Comput. Program., $37(1-3): 67-111,2000$.

[19] N. R. Krishnaswami and N. Benton. A semantic model for graphical user interfaces. In ICFP, pages 45-57, 2011.

[20] S. Lindley, P. Wadler, and J. Yallop. Idioms are oblivious, arrows are meticulous, monads are promiscuous. In MSFP, pages 97-117, 2008.

[21] P. Ljunglöf. Pure functional parsing - an advanced tutorial. Master's thesis, Chalmers, 2002.

[22] C. McBride. Faking it: Simulating dependent types in Haskell. J. Funct. Program., 12(4-5):375-392, 2002.

[23] C. McBride and R. Paterson. Applicative programming with effects. J. Funct. Program., 18:1-13, January 2008.

[24] M. Might, D. Darais, and D. Spiewak. Parsing with derivatives: a functional pearl. In ICFP, pages 189-195, 2011.

[25] B. C. Oliveira and W. R. Cook. Functional programming with structured graphs. In ICFP, pages 77-88, 2012.

[26] B. C. Oliveira and A. Löh. Abstract syntax graphs for domain specific languages. In PEPM, 2013.

[27] R. Paterson. A new notation for arrows. In ICFP, pages 229-240, 2001.

[28] J. Peterson, P. Hudak, and C. Elliott. Lambda in Motion: Controlling Robots with Haskell. In PADL, pages 91-105, 1999.

[29] S. Peyton Jones, D. Vytiniotis, S. Weirich, and G. Washburn. Simple unification-based type inference for GADTs. In ICFP, pages 50-61, 2006.

[30] M. Sulzmann, M. M. T. Chakravarty, S. P. Jones, and K. Donnelly. System F with type equality coercions. In TLDI, pages 53-66, 2007.

[31] S. D. Swierstra and L. Duponcheel. Deterministic, error-correcting combinator parsing. In AFP, pages 184-207, 1996.

[32] J. Voigtländer. Free theorems involving type constructor classes: functional pearl. In ICFP, pages 173-184, 2009.

[33] D. Vytiniotis, S. Peyton Jones, T. Schrijvers, and M. Sulzmann. OutsideIn (X) Modular type inference with local assumptions. J. Funct. Program., 1(1):1-80, 2011.

[34] P. Wadler. Theorems for free! In FPLCA, pages 347-359, 1989.

[35] G. Washburn and S. Weirich. Boxes go bananas: Encoding higherorder abstract syntax with parametric polymorphism. In ICFP, pages 249-262, 2003.

[36] G. Winskel. The formal semantics of programming languages: an introduction. MIT Press, 1993. 